\title{
Advances in diagnostics and therapy of systemic amyloidoses
}

\author{
Krzysztof Jamroziak ${ }^{1,2}$, Bartosz Puła ${ }^{1}$ \\ ${ }^{1}$ Department of Hematology, Institute of Hematology and Transfusion Medicine, Warsaw, Poland \\ ${ }^{2}$ Department of Lymphoma, Maria Skłodowska-Curie National Research Institute of Oncology, Warsaw, Poland
}

For decades therapeutical options to alter the clinical course of systemic amyloidoses have been very limited. However, recent progress in understanding the pathogenesis of these rare disorders have led to the development of novel diagnostic tools and targeted therapies. Here, we briefly review the most important advances in this challenging and interdisciplinary field.

Amyloidoses constitute a heterogeneous group of rare diseases characterized by local or systemic extracellular formation of insoluble amyloid deposits in tissues leading to organ dysfunctions [1]. Tissue deposits are formed by stable and non-branching amyloid fibers resulting from misfolding of different precursor proteins characterized by a beta-sheet structure. The above features of amyloid are common to all amyloidoses regardless of etiology [1]. The type of the precursor protein is the base for nomenclature of amyloidoses developed by the International Society of Amyloidosis (ISA) [1]. Until now 36 amyloidogenic proteins associated with human diseases have been identified including 17 proteins known to cause systemic amyloidoses [2]. The most common types are immunoglobulin light chain amyloidosis (AL amyloidosis), transthyretin amyloidoses (ATTR amyloidosis) comprising wild-type (wtATTR) and hereditary or mutated type (hATTR), and reactive amyloidosis (AA amyloidosis) [1].

The diagnosis of amyloidosis relies on positive staining of tissue deposits with Congo Red that results in characteristic green, yellow or orange tint under polarized light [1]. This test does not distinguish between different types of the disease, thus additional typing is necessary in majority of cases. Unfortunately, access to the gold standard method of amyloid typing using mass spectrometry is very limited, while immunohistochemistry when used by an experienced pathologist may diagnose only up to $70 \%$ of patients [1]. Interestingly, it was found that bone radiotracers, such as $99 \mathrm{mTC}$-DPD or Tc-99m-PYP, bind heart amyloid in ATTR amyloidosis, but not in AL amyloidosis [3]. This observation led to development of non-invasive scintigraphy/SPECT-based typing algorithms, particularly useful to distinguish between isolated cardiac AL amyloidosis and ATTR in the context of monoclonal gammopathy [3]. Furthermore, rapid progress of genetic testing, incorporating next-generation sequencing (NGS) methods, has facilitated screening for hATTR and other extremely rare inherited types of amyloidosis.

From academic perspective treatment of amyloidosis could be divided into two types of strategies: therapies preventing amyloid formation and directed against stable amyloid deposits. Significant progress has been recently achieved concerning the first type of strategies in two most common systemic amyloidoses: AL and ATTR.

Amyloid precursors in AL amyloidosis are monoclonal immunoglobulin light chains produced by clonal plasma cells [4]. Unlike multiple myeloma (MM), the end-organ symptoms in AL amyloidosis are the consequence of the capacity of these light chains to form amyloid deposits, most typically in the heart and kidney [4]. Treatment of $\mathrm{AL}$ amyloidosis is based on MM-type chemotherapy; however, regimen type and intensity need to be individually adjusted due to significantly higher risk of toxicity [4]. An important step forward has been a recent introduction of anti-CD38 monoclonal antibody daratumumab to the treatment armamentarium against $A L$ amyloidosis following its promising outcomes in MM [4].

The phase 3 ANDROMEDA study has evaluated daratumumab-CyBorD (cyclophosphamide, bortezomib, dexamethasone) combination in newly diagnosed $\mathrm{AL}$ amyloidosis [5]. Early results of the trial demonstrated robust hematologic and organ responses without important safety concerns, making it very likely that daratumumab will soon become the first drug approved for $\mathrm{AL}$ amyloidosis [5]. 
Even more spectacular advances have been recently seen in regard to ATTR amyloidosis [6-8]. These novel strategies can be active against both wtATTR (cardiomyopathy) and less frequent hATTR (heterogeneous presentations, mainly neuropathy and cardiomyopathy). Deposition of transthyretin in organs including myocardium occurs when wild-type or mutant transthyretin becomes unstable and misfolds. Tafamidis is a transthyretin stabilizer that prevents transthyretin tetramer dissociation and amyloidogenesis [6]. In a multicenter, double-blind, placebo-controlled, phase 3 ATTR-ACT trial, tafamidis has been tested against placebo in 441 patients with transthyretin amyloid cardiomyopathy with positive results [6]. In the primary analysis, allcause mortality and rates of cardiovascular-related hospitalizations were significantly lower among the patients who received tafamidis [6].

Furthermore, recent development of two novel transthyretin synthesis inhibitors, patisiran and inotersen, may further improve the prognosis in ATTR amyloidosis [7, 8]. Patisiran is a double-stranded small interfering RNA which showed remarkable results in the phase 3 APOLLO study [7]. Patients receiving this compound compared to placebo showed improvement in their sensory, motor and autonomic neuropathy scores as well as nutritional status. Interestingly, patisiran led also to cardiological improvement in patients with confirmed cardiomyopathy [7].

The phase 3 NEURO-TTR study confirmed the clinical utility of inotersen, an anti-sense oligonucleotide targeting transthyretin synthesis [8]. Treatment with inotersen led to neurological improvement within 15 months of therapy, however caution is advised in the course of the therapy due to drug related glomerulonephritis and thrombocytopenia [8].

In AA amyloidosis the amyloid precursor protein is serum amyloid A (SAA) oversecreted by the liver in response to cytokine stimulation, mainly interleukin 6 [2, 9]. This occurs primarily during long-term inflammatory processes such as chronic autoimmune diseases, particularly rheumatoid arthritis and systemic lupus erythematosus, chronic infections and cancer [9]. Interestingly, although specific therapies are not yet available, in recent decades AA amyloidosis have become significantly less frequent probably due to more efficacious treatment of underlying disorders [9].

In contrast, all potential therapies directed against formed amyloid aggregates had so far failed to demonstrate clinical benefit in phase 3 studies. This has happened recently to NEOD001 (birtamimab), a humanized IgG1 antibody designed to directly stimulate neutralization of toxic aggregates of misfolded light chains via phagocytosis [10]. Despite promising early clinical re- sults, the phase 3 VITALE evaluating NEOD1 added to standard of care therapy was terminated early due to inefficacy [10]. Nevertheless, other similar strategies, including CAEL-101, are being actively developed.

It should be underlined that beside novel active therapies, proper management of such rare and complex diseases as amyloidoses requires both general awareness and specialized centers with interdisciplinary approach. In response to these needs, we initiated the Polish Amyloidosis Network (www.amyloidosis.org.pl) conceived as a platform for educational, clinical and scientific projects aimed at improving of diagnostics and care of amyloidosis in Poland.

The authors declare no conflict of interest.

\section{References}

1. Wechalekar AD, Gillmore JD, Hawkins PN. Systemic amyloidosis. Lancet 2016; 387: 2641-2654, DOI: 10.1016/S0140-6736 (15)01274-X.

2. Benson MD, Buxbaum JN, Eisenberg DS, et al. Amyloid nomenclature 2018: recommendations by the International Society of Amyloidosis (ISA) nomenclature committee. Amyloid 2018; 25: 215-219, DOI: 10.1080/13506129.2018.1549825.

3. Aljaroudi WA, Desai MY, Tang WH, et al. Role of imaging in the diagnosis and management of patients with cardiac amyloidosis: state of the art review and focus on emerging nuclear techniques. J Nucl Cardiol 2014; 21: 271-283.

4. Jamroziak K, Milani P, Puła B, et al. Diagnostyka i leczenie amyloidozy AL. Hematologia 2018; 9: 181-195, DOI: 10.5603/ Hem.2018.0024.

5. Palladini G, Kastritis E, Maurer MS, et al. Daratumumab plus CyBorD for patients with newly diagnosed AL amyloidosis: safety run-in results of ANDROMEDA. Blood 2020; 136: 71-80, DOI: 10.1182/blood.2019004460.

6. Maurer MS, Schwartz JH, Gundapaneni B, et al., for the ATTRACT Study Investigators. Tafamidis Treatment for Patients with Transthyretin Amyloid Cardiomyopathy. N Engl J Med 2018; 379: 1007-1016, DOI: 10.1056/NEJMoa1805689.

7. Adams D, Gonzalez-Duarte A, O'Riordan WD, et al. Patisiran, an RNAi Therapeutic, for Hereditary Transthyretin Amyloidosis. N Engl J Med 2018; 379: 11-21, DOI: 10.1056/NEJMoa1716153.

8. Benson MD, Waddington-Cruz M, Berk JL, et al. Inotersen Treatment for Patients with Hereditary Transthyretin Amyloidosis. N Engl J Med 2018; 379: 22-31, DOI: 10.1056/NEJMoa1716793.

9. Westermark $G$, Fändrich $M$, Westermark P. AA amyloidosis: pathogenesis and targeted therapy. Annu Rev Pathol 2015; 10 : 321-344, DOI: 10.1146/annurev-pathol-020712-163913.

10. Gertz MA, Cohen AD, Comenzo RL, et al. Results of the Phase 3 VITAL Study of NEOD001 (Birtamimab) Plus Standard of Care in Patients with Light Chain (AL) Amyloidosis Suggest Survival Benefit for Mayo Stage IV Patients. Blood 2019; 134: 31663166, DOI: 10.1182/blood-2019-124482. 